\title{
Choice of Partners in Multiple Two-Person Prisoner's Dilemma Games: An Experimental Study*
}

\author{
Esther Hauk ${ }^{\dagger}$ and Rosemarie Nage ${ }^{\frac{1}{*}}$ \\ Department of Economics and Business \\ Universitat Pompeu Fabra \\ Ramon Trias Fargas 25-27 \\ 08005 Barcelona (Spain)
}

July 2000

\begin{abstract}
We examine the effect of unilateral and mutual partner selection in the context of prisoner's dilemmas experimentally. Subjects play simultaneously several finitely repeated two-person prisoner's dilemma games. We find that unilateral choice is the best system. It leads to low defection and fewer singles than with mutual choice. Furthermore, with the unilateral choice setup we are able to show that intending defectors are more likely to try to avoid a match than intending cooperators. We compare our results of multiple games with single game PD-experiments and find no difference in aggregate behavior. Hence the multiple game technique is robust and might therefore be an important tool in the future for testing the use of mixed strategies.
\end{abstract}

Keywords: Prisoner's dilemma, partner selection, experiments, multiple games JEL classification numbers: C72, C91

We thank Antonio Cabrales and Josep Colomer for helpful comments. We also thank Andreoni and Miller for letting us use their experimental data. Esther Hauk acknowledges financial support of DGES under project PB96-0118 and from the Generalitat de Catalunya project 1999SGR00157. Rosemarie Nagel acknowledges financial support of DGES under project PB98-1076, and the EU-TMR Research Network ENDEAR (FMRX-CT98-0238).

屯-mail: esther.hauk@econ.upf.es.

e-mail:rosemarie.nagel@econ.upf.es 


\section{Introduction}

The central feature of the prisoner's dilemma (PD) is that each player creates and consumes externalities: the externality created is positive, if the player cooperates and negative, if the player defects. The crux of the standard PD is that no player can escape this externality. While many situations that have been formalized by the PD certainly have this lock-in feature, there are also many naturally occurring situations in which escape routes arise. Tiebout (1956) already pointed at the possibility of "voting with your feet" to the provision of public goods in metropolitan areas. Hirschman (1970) interprets cooperation as "loyalty" and defection as "voice" in the political context. He conceives "exit" as an alternative action to loyalty without paying the cost of voice. Orbell and Dawes (1993) use university research as a metaphor for PD games with no obligation to play. Firms may choose their suppliers. The firm-supplier relationship is cooperative if firms pay on time and suppliers deliver punctually. People choose their friends. Even criminals choose their partners in crime.

In recent years the game theoretical literature on partner selection has mushroomed (see e.g. Ashlock et al. (1996), Ghosh and Ray (1996), Hauk (2000), Orbell, Schwartz-Shea and Simmons (1984), Orbell and Dawes (1991, 1993), Peck (1993), Schluessler (1989), Stanley et al. (1994), Tesfatsion (1997) and Vanberg and Congelton (1992)). While these papers examine different types of partner selection mechanisms they all use MUTUAL CHOICE: the PD game is played between two players if and only if both players mutually agree to play the game. In the present paper we additionally study UNILATERAL CHOICE: the PD game has to be played if at least one partner chooses to do so.

Marriage is an example of a repeated PD game which historically has passed from lockin to unilateral and later to mutual choice. Until the 19th century parents negotiated and arranged their children's weddings in most countries in Asia and in Europe: the spouses were locked in to play the "game". It seems that, in general, they learned to adapt and even to love each other, producing long lasting, moderately happy families. In more recent times, the man unilaterally asked the girl's parents for her hand, while the girl did not have much room for refusal. Finally, today with the "liberation of women", the choice is mutual and divorce is legal. While there seem to be fewer domestic battles between husband and wife, the number of stable couples is lower than in the past and the number of singles has increased. This suggests that partner choice increases the level of cooperation within the games which are actually played, but also leads to fewer games.

In this paper we study the effect of unilateral and mutual choice of partners in finitely repeated $\mathrm{PD}$ games. We will compare three experimental treatments: 1.) the lock-in setup, i.e. the classical PD game with no possibility to choose the partner, 2.) the PD 
game with unilateral choice and 3.) the PD game with mutual choice. ${ }^{1}$ Partner choice is modeled by adding a "matching" stage to the classical PD game; in this matching stage each player is given an exit opportunity with a sure payoff (as in Orbell, SchwartzShea and Simmons (1984), Orbell and Dawes (1991, 1993), Morikawa, Orbell and Runde (1995), Orbell, Runde and Morikawa (1996) Hauk (1997,1999)).

In the experiments, we are only interested in the situation where the exit option yields higher payoffs than mutual defection. Hence, to remain single is better than to be paired with a defector. This implies that the decision between entering or not will be critically influenced by a player's prediction about a potential partner's intention how to play the PD game. Whatever one's own intentions between cooperation and defection, it is worthwhile to enter the $\mathrm{PD}$ game only if the probability to encounter cooperation is high. Indeed, we observe that defection levels are much lower in the choice treatments than in the lock-in PD. Moreover, (conditional) cooperation levels, given a PD-match, are higher in the unilateral and mutual choice setup than in the standard lock-in PD game. Arranged couples are less happy than voluntary couples.

However, when examining the ratio of cooperation levels out of all possible choices (including exit-choices), these unconditional cooperation levels are indistinguishable in the classical PD game and the unilateral choice setup, but are significantly lower in the game with mutual choice. At the same time, unconditional defection levels are much lower in the choice setups (lowest under mutual choice). In terms of our marriage example this means that under mutual choice the number of happily married couples in the total population is lowest, but so is the number of unhappy couples. Only strongly cooperative relationships will be long lasting. If one's partner decides to split, there is no chance to convince him to continue by showing cooperativeness. The latter is always possible under unilateral choice resulting in higher unconditional cooperation levels. ${ }^{2}$

Our novel unilateral choice setup allows us to study what type of player chooses the outside option. In this setup a subject who chooses to exit might be forced to play the game by his partner. Under these circumstances the exit player defects $81 \%$ in the PDgame. In contrast, an entry player only defects in $24 \%$ of the games played. Hence, intending defectors are more prone to avoid the game than intending cooperators.

Orbell et al. (1984) and Orbell and Dawes (1993) already tried to test this hypothesis (intending defectors exit more frequently than intending cooperators) in experiments on one-shot PD games. However, they used the mutual choice setup, in which intentions in the PD-phase cannot be observed if exit is chosen. Orbell et al. (1984) circumvent

\footnotetext{
${ }^{1}$ The data for the lock-in treatment and the mutual choice treatment is taken from Hauk (1999).

${ }^{2}$ Our unilateral choice setup is symmetric and therefore differs from the historical unilateral choice in marriage. In our setup both players have equal rights.
} 
this problem by "turning around" the structure of the game. First subjects are asked to choose between cooperation and defection and then have to decide whether or not to enter the game. The drawback of this design is that for someone who decides to exit, the PD-choice is inconsequential; subjects who exit might therefore lack incentives to reveal their true intention in the PD-phase.

Orbell and Dawes (1993) use a different approach to measure intentions in unplayed PD games. They extrapolate the number of intending defectors and cooperators when exit occurred from the number of defectors and cooperators in an experiment on the classical one-shot PD game. Apart from using a different set of subjects for the classical lock-in PD and the mutual choice PD, the two games differ and a player who cooperates in one might want to defect in the other, which casts serious doubts on this method. On the contrary, our unilateral choice setup offers a direct test for cooperative or defective intentions when opting out.

Besides the new unilateral choice setup, we also use a rather new technique of multiple plays within a period. In each treatment each player plays simultanously several 2-person supergames at the same time and gets the opportunity to repeat supergames. As in Hauk (1999), each player has to make separate decisions for several partners in the same period. Given the wide variety of situations that have a PD structure, it is reasonable to assume that in real life we usually play several PD games with different partners at the same time. The following example nicely fits our setups: in many universities or schools the same course is offered by different teachers. Some universities require coordination between the professors and thus they have to play a game that resembles a PD-game. Other universities encourage coordination while others leave it to the professors to coordinate or not, in which case unilateral or mutual agreements are necessary to enter a PD game. Moreover, professors usually teach several different courses, hence they play multiple simultaneous PD games with different partners. Additional games occur outside their professional sphere (e.g. with their friends and sentimental partner).

With the help of the multiple game technique we can see whether a player is a pure defector or a pure cooperator. Most subjects do implement different choices with different game partners even in the first period of a supergame where everybody is the same ex ante. Probabilistic behavior is common.

But are our results robust to the multiple game method? Could it not be that the probabilistic behavior is directly caused by the multiple games, e.g. because players learn or experiment across games? When comparing our classical PD setup of multiple matching to single game experiments (using the data set by Andreoni and Miller (1993)), we find that there is no difference in aggregate behavior over time. This strongly suggests robustness. This is an important result, since it implies that the multiple game technique 
might be useful for running experiments in which probabilistic behavior might matter, e.g. games with a unique mixed equilibrium. This technique would offer subjects a natural way to implement probabilistic choices without having to explain the concept of probability.

The robustness of the multiple game technique is also confirmed in experiments on the dictator game due to Bolton, Katok and Zwick (1998). In their experiment a dictator is matched (i) with only one recipient and (ii) with 10 recipients. In both treatments he has to divide the same amount of money. They find that the distributions of the total gift are statistically indistinguishable. As in our experiments the multiple game treatment reveals probabilistic behavior: in the 10 recipient treatment, the dictator does not give the same amount to every recipient.

Ochs (1995) uses also multiple game technique for $2 \times 2$ normal games with a unique mixed equilibrium. The main difference is that his subjects play 10 simultaneous games against the same opponent within a period and then switch the opponent. Playing simultaneous games with the same opponent would not have made sense in the PD-context, so we believe that our method is useful for more general contexts.

The remainder of the paper is organized as follows. In the next section we describe the different games which are studied experimentally. Section 3 states the experimental design, section 4 discusses the experimental results and section 5 concludes.

\section{The games under consideration}

\subsection{The three setups}

We add two design features to the classical PD-supergame. The first feature contains two ways of partner selection before a PD-game is played. The second feature is that each player is matched with multiple partners at the same time, and each player has to make separate decisions for each partner. Thus we study behavior in series of finitely repeated 2-PERSON prisoner's dilemma supergames with multiple partners with or without pre-partner selection.

In the two pre-partner selection setups, every period consists of at most two phases: In the first phase a player has to decide for all potential partners, separately, whether he wants to enter to play a PD-game or whether he wants to exit. In the potential second phase the PD-game is played. All in all we distinguish three setups:

1. the prisoner's dilemma game (without a pre-partner selection phase). We will call this setup lock-in $P D($-setup).

2. the prisoner's dilemma game with a pre-partner selection phase ("selection setups") such that 
(a) if at least one of the two potential partners agrees to enter they go to the second phase and play a PD game. If both exit, the game ends. We will call this setup unilateral choice $P D($-setup)

(b) if two potential partners mutually agree to enter they go to the second phase and play a PD-game. In the other cases they do not enter the second phase and the game ends. We will call this setup mutual choice $P D(-$ setup $) .^{3}$

A single game setup (each player has only one partner within a finitely repeated 2person PD game) will serve as our control experiment. This setup is taken from Andreoni and Miller (1993).

\subsection{The payoff matrices}

In all setups the same two-person prisoner's dilemma game with the following bimatrix of the one-shot game is implemented. ${ }^{4}$

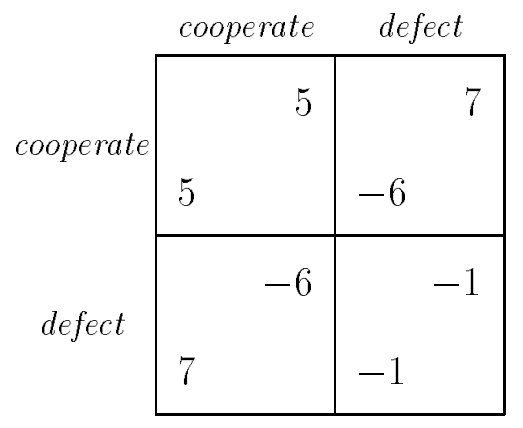

\section{Prisoner's Dilemma}

The difference between the two choice setups is illustrated in the following figure. The bimatrices present the reduced normal forms of the one-shot game of the unilateral choice PD and of the mutual choice PD. PD in a cell of the matrices means that the prisoner's dilemma game is played and the payoffs depend on the decisions in that game. If there is no second phase then both players get the outside-option payoff of zero which is higher than the payoff of mutual defection in the second phase. (The zero payoff is chosen, since we know from prospect theory that it serves as a status quo from which people evaluate their gains and losses and does not affect the evaluation of their final wealth.)

\footnotetext{
${ }^{3}$ Our data for the choice setup is taken from Hauk (1999) using repeated games.This setup is also studied experimentally by Orbell et al. (1984) and Orbell and Dawes (1993) in one-shot games.

${ }^{4}$ During the experiment defection was coded by $a$ and cooperation by $b$.
} 


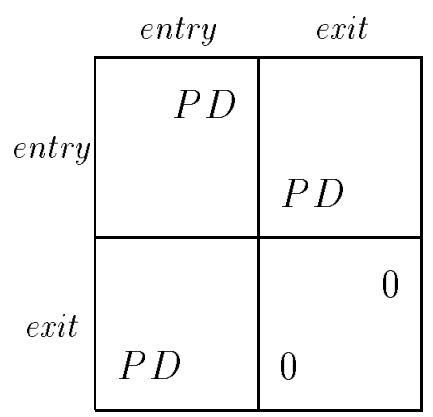

unilateral choice

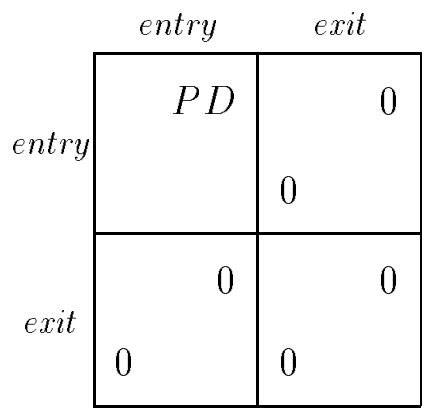

mutual choice

\subsection{Game theoretic analysis}

\subsubsection{The finitely repeated PD-supergame}

In the finitely repeated prisoner's dilemma game, there is only one Nash-equilibrium in which both players choose their strictly dominant strategy: defection in every period.

\subsubsection{The finitely repeated unilateral choice PD-supergame}

In the unilateral choice PD, a player can force the potential partner to enter the PD-phase but also can be forced to play the PD game. In this setup, if a player enters, the other player's participation constraint becomes irrelevant, resulting in several Nash equilibria in the one-shot game, two of which are subgame perfect, namely:

1. both players exit and defect in the unreached game

2. both players enter and defect.

Both equilibria are subgame perfect equilibria of the finitely repeated choice PD. Additional equilibria exist, some of which can sustain cooperation. In particular, in a 10 period supergame mutual cooperation until period 7 (inclusive) followed by mutual exit (and defect in the unreached game) until the end of the supergame can by sustained by the following out-of equilibrium beliefs: defection in the cooperative phase is going to be punished by entering and defecting for ever.

\subsubsection{The finitely repeated mutual choice PD-supergame}

In the mutual choice PD, players are able to avoid unwanted matches and obtain a secure payoff of zero. If one player chooses the outside option, the other player's participation decision becomes irrelevant. Hence, even the one-shot game has several Nash equilibria. These equilibria are characterized by at least one player opting out (and if they are 
subgame perfect by the intention to defect in the unreached game). Given that these multiple equilibria of the one-shot game all have the same outcome (outside option), the subgame perfect equilibria (SPE) of the finitely repeated game also lead to the outside option outcome.

\section{$3 \quad$ Experimental design}

Subjects were recruited from the first year undergraduate student population of the economics, business and humanity faculties of Universitat Pompeu Fabra, Barcelona. Every subject participated in only one session. The students met in the experimental laboratory LEEX. The experiment was computerized. Written instructions were read out aloud (see appendix).

For each of the three setups, lock-in PD, unilateral choice PD and mutual choice PD, 3 sessions were run with 7 subjects in each. In every period, each player had 6 partners. This means that in every period each subject played 6 simultaneous two-person games. Subjects made their choices period by period and for each of the 6 simultaneous twoperson games separately. A supergame lasted 10 periods. In total every player played 6 partners $\mathrm{x} 10$ periods x 10 supergames. Within a supergame each subject was identified by one player number $1,2, \ldots$ or 7 and was introduced by that number to each of his partners. In the next supergame the computer randomly re-assigned subjects' identities. This way a player received information about a particular partner for every period within the same supergame, but did not know what that partner had done in previous supergames against him.

After each period, each player was informed about his payoff and the partner's payoff of each match, and also about the partner's choice if the PD-game was played. Information about the pre-partner selection phase was reduced to whether or not the second phase was entered but no explicit information about the partner's decision in the first phase was given. The entire history of past play was available to subjects at any point in time.

\subsection{Payment}

Subjects were given a starting capital of 1000 Pesetas to ensure them against bankruptcy since some of the payoffs in the PD game were negative. Each player was additionally paid by what he earned in two randomly chosen ${ }^{5}$ supergames $(2 \times 6 \times 10$ interactions). Each point of the matrices was converted into 15 Pesetas. This kind of payment seemed sensible

\footnotetext{
${ }^{5}$ Each student draw a number from each of two urns which contained supergame 1 to 5 and 6 to 10 , respectively.
} 
since each subject participated in a total of $6 \times 10 \times 10=600$ interactions. A payoff of each single interaction would have required 1 point to be converted into 1 Peseta to equate the average payoff of 1500 Pesetas an hour. We believe that this small conversion rate would not have given subjects sufficient incentives to take every single decision seriously. All subjects were paid privately.

\subsection{Control Experiment}

The single-game control experiment is taken from Andreoni and Miller (1993). We use their data of the so-called partners condition: in this setup 14 subjects were randomly paired to play a 10 -periods repeated prisoner's dilemma game with their partner. ${ }^{6}$ They were then randomly rematched for a total of 2010 -period games. The information structure of Andreoni and Miller (1993) was essentially the same as for our lock-in PD-setup. Since our sessions lasted for 10 supergames we only use the data of their first 10 supergames. We will refer to the control setup as A\&M.

\section{$4 \quad$ Results}

In the present experiments we compare setups in which playing the $\mathrm{PD}$ game can be avoided (unilateral and mutual choice PD) to a setup where the PD game has to be played (lock-in PD). How much cooperation or defection occurs can be measured in two ways: the number of cooperative (defective) choices (i) out of all possible choices (including exit) or (ii) in PD games that are actually played. We will refer to these two different measures as (i) unconditional and (ii) conditional cooperation (defection) levels

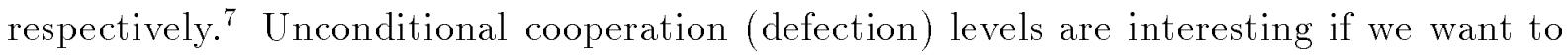
study the total amount of cooperation (defection) that arises in the population, while conditional cooperation (defection) levels tell us how much cooperation (defection) is chosen within those PD games that are actually played. Considering our marriage analogy, unconditional cooperation levels tell us the percentage of the population that is happily married, while conditional cooperation levels tells us the percentage of happily married couples.

Table 1 reports conditional and unconditional cooperation levels, defection levels and the proportion of cases in which no match occurred for the lock-in PD, the unilateral choice PD, the mutual choice PD and our control setup A\&M. The data is aggregated

\footnotetext{
${ }^{6}$ The following payoff matrix was used: payoffs 7,7 for $c, c ; 0,12$ for $c, d$ amd 4,4 for $d, d$.

${ }^{7}$ In the lock-in PD and in A\&M these two measures coincide since exit is not possible.
} 
over all subjects, periods and sessions. Table 1 therefore gives a first impression of the different outcomes of no choice (lock-in), unilateral and mutual choice.

\begin{tabular}{|c|c|c|c|c|}
\hline & & cooperation & defection & no match \\
\hline \multirow{4}{*}{ unconditional } & $\mathrm{A} \& \mathrm{M}$ & 0.38 & 0.62 & - \\
\hline & lock-in & 0.36 & 0.64 & - \\
\hline & unilateral & 0.38 & 0.26 & 0.36 \\
\hline & mutual & 0.25 & 0.20 & 0.54 \\
\hline \multirow[t]{2}{*}{ conditional } & unilateral & 0.58 & 0.42 & \\
\hline & mutual & 0.51 & 0.49 & \\
\hline
\end{tabular}

Table 1: summary data of cooperation, defection and no match in all treatments

This first impression can be stated as follows:

1. Unconditional and conditional defection is much higher in lock-in treatments than under choice treatments.

2. Conditional cooperation levels are higher, if partner choice is possible, i.e. with partner choice married couples are happier. However, unconditional cooperation levels are lower if choice is mutual (25\%) than under unilateral choice (38\%) or no choice $(36 \%)$, i.e. there are fewer happily married couples in the total population (including singles) when choice is mutual.

3. There are more singles under mutual choice (54\%) than under unilateral choice $(36 \%)$.

4. Cooperation and defection levels in our lock-in setup with multiple games are similar to the lock-in setup with a single game A\&M taken from Andreoni and Miller (1993).

While mutual choice minimizes the number of unhappily married couples, it also reduces the number of happily married couples in the total population: it seems that the couple splits if things do not work out immediately (i.e. if cooperation does not arise). Under unilateral choice the couple can only split if both agree to do so, which slightly increases the number of unhappily married couples but also increases the number of happily married couples. Hence, there can be some benefit of being locked in. However, pure lock-in is clearly worse than unilateral or mutual choice since defection is significantly higher. 
Another possible application of this framework can be the analysis of some citizens' behavior under a government in conflict with a neighbor state, as, for instance, the two Germanies during the period 1949-1990 and the Cuba and the United States governments since 1959. If an authoritarian government chooses to close its borders unilaterally, trying to preclude its citizens' possibility to choose which government to live with (as the East German and Cuban governments did), it can reduce the number of people choosing exit, i.e. emigrating to the neighbor country. But it can also increase the level of loyalty to the government: in both lock-in and unilateral closing of borders, some people adapt and learn to cooperate with the government. In contrast, with mutually open borders, the level of exit is higher and loyalty is lower. (For the German case, see Hirschman (1993); for Cuba, see Colomer (2000)).

\subsection{Behavior over time}

In the following subsections we confirm the above picture in a more detailed analysis looking at the time structure. Figure $1 \mathrm{a}, \mathrm{b}, \mathrm{c}$ shows relative frequencies of unconditional cooperation, defection and no match in each of the 100 periods, separately for each treatment. The vertical dividing lines in each graph separate one supergame from the other. Figure 1a presents the relative frequency of cooperation of our lock-in PD and of the Andreoni-Miller PD-setup. Figure 1b,c shows the relative frequencies of cooperation (gray area), defection (white area) and no-matches (dotted area) for the mutual choice $\mathrm{PD}$ and the unilateral choice $\mathrm{PD}$, respectively.

\subsubsection{Unconditional Defection Levels}

Observation 1 (Unconditional) defection levels are much higher in the lock-in treatment than under partner choice.

Equal unconditional defection levels are strongy rejected by the Wilcoxon-MannWhitney test. ${ }^{8}$ The p-value for the hypothesis of equal defection levels between unilateral choice and lock-in and mutual choice and lock-in is $p=0.0286$ in both cases. Observation 1 is also confirmed in Figures $1 \mathrm{a}, \mathrm{c}, \mathrm{b}$. The areas representing unconditional defection levels in each period (white areas) are much bigger in the lock-in treatment (and in A\&M) than in the choice treatments.

\footnotetext{
${ }^{8}$ For the Wilcoxon-Mann-Whitney test we have 3 observations (sessions) for each of the choice setups and 4 observations (sessions) for the lock-in PD. The latter includes the Andreoni-Miller (1993) data since it is very similar to our lock-in data.
} 
Lock-in and A\&M, pooled over all sessions

(relative frequencies of cooperation)
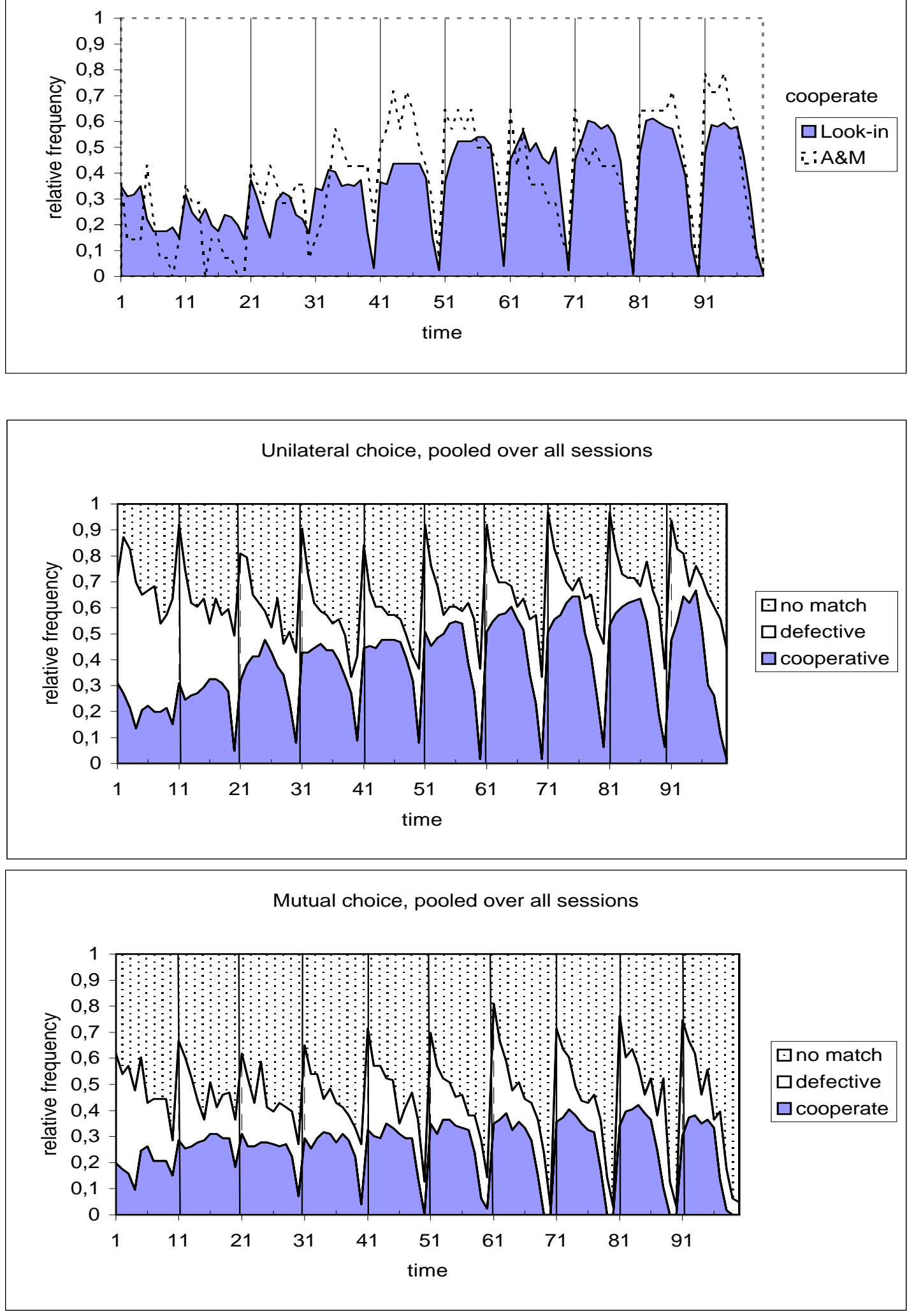

Figure 1: Relative frequencies of unconditional per period cooperation, defection and no match 


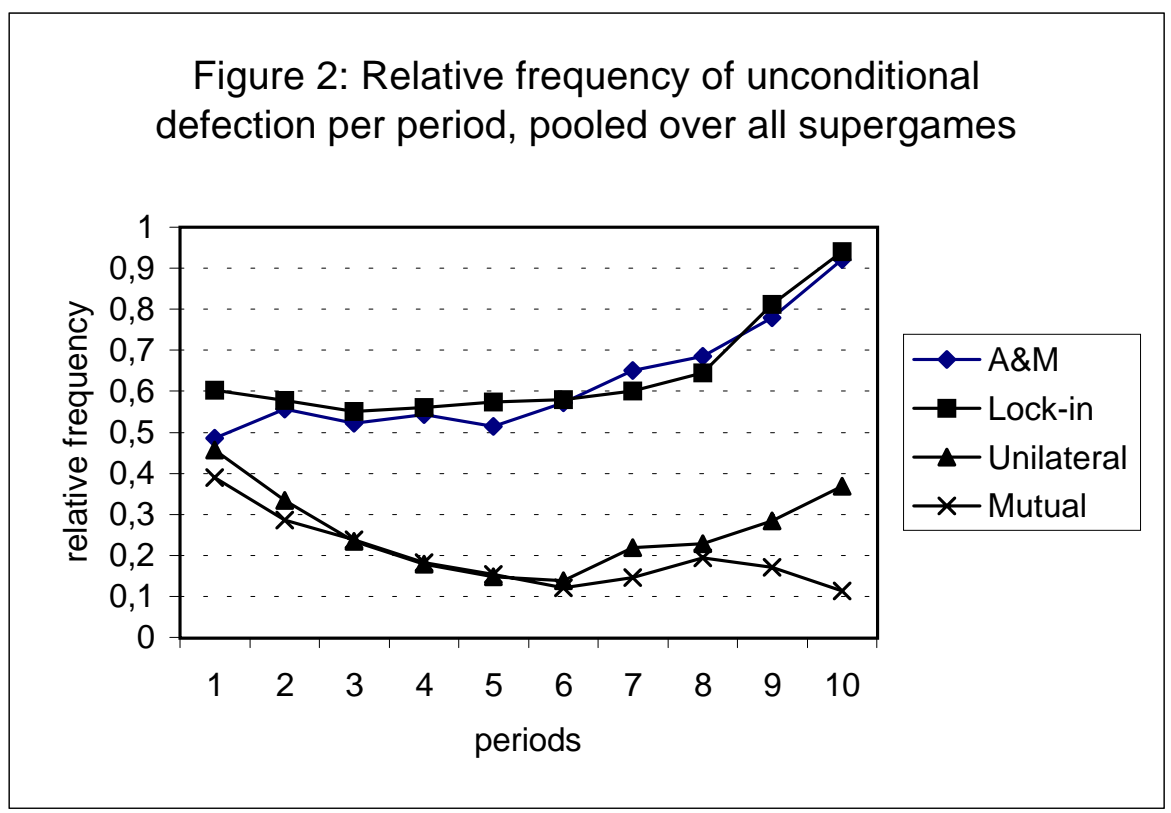

Figure 2: Average Unconditional Defection per Period.

Figure 2 shows unconditional defection levels per period averaged over all supergames. The figure reveals that unconditional defection levels are consistently higher in the lock-in setup. ${ }^{9}$ Under partner choice people are less unhappy. The difference in defection levels is smallest in the first period and increases over time. In subsection 4.1.3 we will see that partner choice reduces defection levels because subjects do not want to play with defectors and (intending) defectors themselves are more likely to exit (see also the no match areas in Figures $1 \mathrm{~b}$ and $\mathrm{c}$ ).

\subsubsection{Cooperation levels}

Although defection levels are clearly highest in the lock-in setup, Figure 1 also reveals that the obligation to play might have some positive effect on cooperation levels. Only under mutual choice a player can never be forced to play the PD game.

\footnotetext{
${ }^{9}$ In the final periods players who previously cooperated stop cooperation because they know that the supergame ends (end-effect). In the lock-in treatment the only possible end-effect behavior is to defect. In the choice treatments players can additionaly choose not to play. Therefore, it is not surprising that defection levels are much higher in lock-in treatments during the endeffect periods. Defection levels are higher during the last periods in the unilateral choice PD than in the mutual choice PD since it is easier to stop playing under mutual choice.
} 
Observation 2 Unconditional cooperation levels are highest in the unilateal choice PD and lowest in the mutual choice PD (see Figures 1a,b,c)

Figures $1 \mathrm{a}$ and $\mathrm{c}$ illustrate that the unconditional cooperation frequencies in the lockin $\mathrm{PD}$ and the unilateral choice $\mathrm{PD}$ are very similar, as well in the first supergames, as in the last supergames. Also the Wilcoxon-Mann-Whitney test gives a relatively high probability, namely $p=0.35$, to the hypothesis of equal unconditional cooperation levels in the lock-in PD and the unilateral choice PD. On the contrary, the unconditional cooperation levels in the mutual choice setup are much lower, which shows the positive effect on cooperation levels of lock-in relationships. The Wilcoxon-Mann-Whitney test leads to a p-value of $p=0.1143$ for a comparison of the lock-in $\mathrm{PD}$ and the mutual choice $\mathrm{PD}$ and to a $p=0.05$ for a comparison between the two pre-selection setups.

Under mutual choice both unconditional cooperation and unconditional defection are lowest. This implies that cooperation is high in those games that are actually played.

Observation 3 Cooperation levels conditional on a match are highest in the unilateral choice-PD and lowest in the lock-in PD.

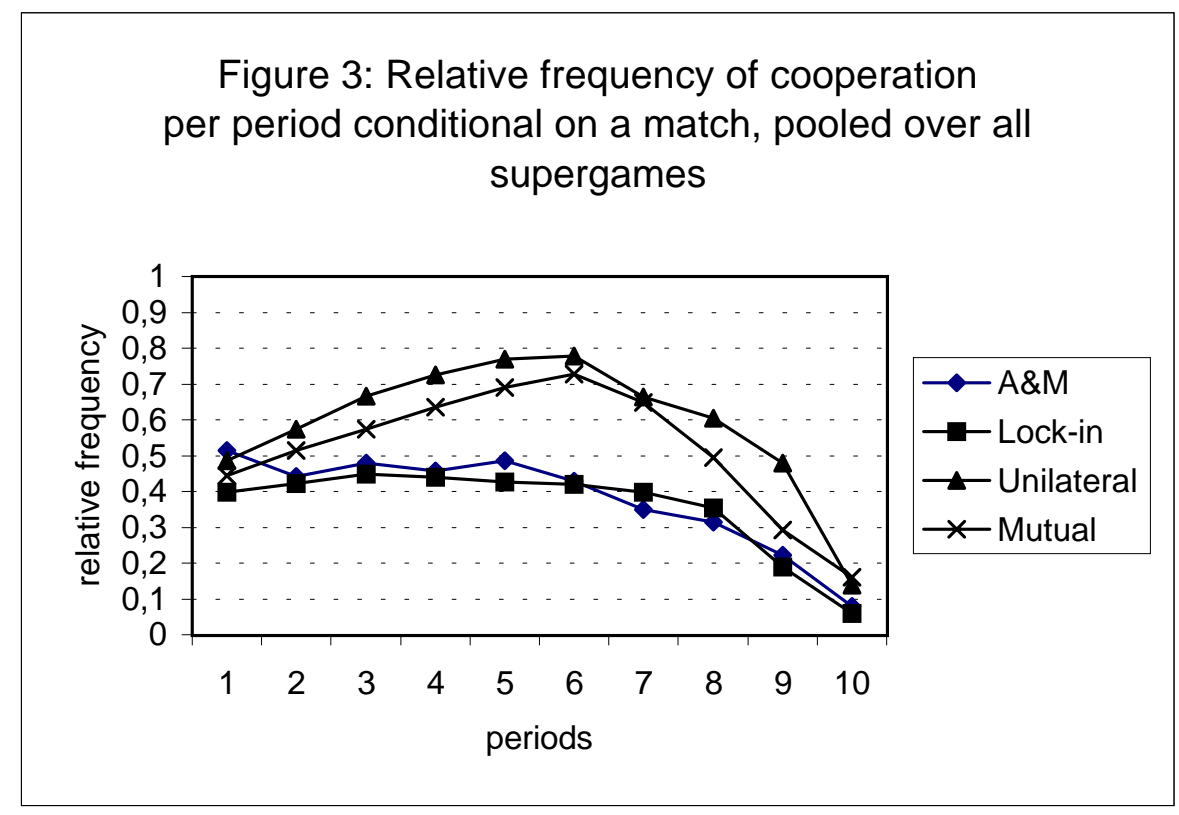

Figure 3: Average Cooperation per Period Conditional on Play.

Figure 3 shows the average cooperation levels (conditional on a PD-match) per period within a supergame, aggregated over all supergames for each treatment, separately. Comparing the average cooperation levels per session between the unilateral choice-PD and 
the lock-in PD, the Wilcoxon-Mann-Whitney test (applied as before) reveals a p-value of $p=0.0286$ for the hypothesis of equal cooperation levels. The p-value for the comparison of the mutual choice setup ( 3 sessions) and the lock-in PD-setup is $p=0.20$ and for the comparison between the unilateral choice and the mutual choice $\mathrm{PD}$ is $p=0.35$.

\subsubsection{Exit}

The similarity of unconditional cooperation levels in the unilateral choice PD and the lockin PD implies that the sum of no-match and defection in the unilateral choice treatments is similar to defection in the lock-in PD-treatment. Subjects seem to choose no-match as an alternative to defection. If this were correct, we should observe defection if a subject who chooses exit in the preselection phase is forced to play in the PD-phase; this might happen in the unilateral choice-PD since only one "entry"-player is needed for a match. Indeed, examining subjects' PD actions in unwanted matches in the unilateral choice-PD reveals:

Observation 4 Intending defectors are more likely to opt out than intending cooperators.

We observe that $81 \%$ of the "exit"-players defect when they are forced to play. After an "in"-choice, on the other hand, only $24 \%$ defect. No-match is indeed an alternative to defection.

A close look at Figure 3 corroborates this interpretation. While in the lock-in PD cooperation rates are rather stable over time, conditional cooperation levels in the preselection setups are hill-shaped, increasing to $80 \%$ in period 5 and 6 . Given that unconditional cooperation levels in the unilateral choice $\mathrm{PD}$ and in the lock-in $\mathrm{PD}$ are very similar, this steep increase in conditional cooperation levels in the unilateral choice PD seems to be due to subjects opting out if cooperation cannot be established after the opening periods of a supergame while in the lock-in PD they defect. Indeed, in the unilateral choice PD a player who experiences mutual defection exits with probability 0.68 . Cooperation against a defector provokes an exit rate of $48 \%$ while defection against a cooperator provokes an exit rate of $49 \%$. After mutual cooperation the exit rate is only $2 \%$. These transition probabilities indicate clearly that exit is caused by expecting defection in the PD game as had been suggested already by Orbell et al. (1993). However, Orbell et al. (1993) only examined the one-shot context with mutual choice in which a player's exit decision could not be conditioned on his personal experience with a specific game partner and the causes for exit could not be observed. Also, they had to speculate how the PD game would have been played in case of exit while our unilateral choice setup allows us to observe the PD choice of an "exit"-player directly. 
A further advantage of our experimental design is that subjects play multiple games. The multiple game technique reveals more about the true intention of each subject than standard single game setups: the experimenter receives more information in each time period and subjects can transmit probabilistic choices. Probabilistic behavior is observed if a subject does not use the same strategy in the same period with identical game partners (same history of play). While it is very likely that the history of play evolves differently with distinct partners, in the first period of each supergame all game partners are identical. To get a better understanding of subjects' true intentions, we therefore examine first period behavior.

\subsection{First period behavior}

The multiple game technique allows us to examine whether initial intentions of a player are pure, i.e. whether he always chooses the same actions in the selection stage and in the PD-stage against all his game partners or whether he mixes, i.e. he only enters some of his 6 possible matches or sometimes defects and sometimes cooperates. As in Hauk (1997, 1999) we observe:

Observation 5 First period behavior is very heterogeneous within a subject and across subjects: mixed types are very common.

In the lock-in PD, $43 \%$ of the cases players mix, $38 \%$ always defect and $19 \%$ always cooperate. In the unilateral choice setup $65 \%$ of the subjects mix in at least one of the stages of the game. $63 \%$ of the subjects mix in the selection stage and $39 \%$ mix in the PD stage. Under mutual choice, $71 \%$ of the subjects mix in at least one of the stages of the game: $30 \%$ mix in the selection stage and $47 \%$ in the PD stage.

Probabilistic behavior might be a way to balance the possible negative payoffs from cooperating against a defector with the high positive payoff from defecting against a cooperator. At the same time, at least some cooperative signals are sent which might lead to a stable cooperative behavior.

\subsection{Robustness of the multiple games technique}

Hauk (1999) has already shown that mixing behavior is not due to learning or experimentation, since it does not disappear with more experience. In order to be sure that our observations on probabilistic behavior are not caused by the multiple game setup we check the robustness of our results by comparing them to a single game setup.

Observation 6 The multiple game technique is robust. 
1. There is no significant difference in aggregate behavior over time between our control experiment (Andreoni and Miller) and the PD-setup. This is immediately seen in Figure $1 \mathrm{a}$ and 2.

2. All treatments share the typical characteristics of finitely repeated single game PDs as studied in Selten and Stoecker (1987):

(a) cooperation levels increase from supergame to supergame.

(b) defection and/or no match increase in the last period(s) from supergame to supergame (endeffect).

Table 2 provides a simple measure for these two observations in our data sets by showing the average cooperation levels in each period within the first five supergames and within the last five supergames, for each treatment separately.

\begin{tabular}{|c|c|c|c|c|c|c|c|c|c|c|c|c|}
\hline \multirow{2}{*}{$\begin{array}{l}\text { setup } \\
\text { period }\end{array}$} & \multicolumn{3}{|c|}{$\begin{array}{l}\text { A\&M } \\
\text { supergames }\end{array}$} & \multicolumn{3}{|c|}{$\begin{array}{l}\text { lock-in } \\
\text { supergames }\end{array}$} & \multicolumn{3}{|c|}{$\begin{array}{l}\text { mutual choice } \\
\text { supergames }\end{array}$} & \multicolumn{3}{|c|}{$\begin{array}{l}\text { unilateral choice } \\
\text { supergames }\end{array}$} \\
\hline & $1-5$ & $6-10$ & all & $1-5$ & $6-10$ & all & $1-5$ & $6-10$ & all & $1-5$ & $6-10$ & all \\
\hline 1 & 0.36 & 0.67 & 0.51 & 0.35 & 0.44 & 0.40 & 0.28 & 0.34 & 0.31 & 0.36 & 0.51 & 0.43 \\
\hline 2 & 0.31 & 0.57 & 0.44 & 0.31 & 0.54 & 0.42 & 0.25 & 0.36 & 0.31 & 0.36 & 0.54 & 0.45 \\
\hline 3 & 0.36 & 0.60 & 0.48 & 0.32 & 0.58 & 0.45 & 0.25 & 0.39 & 0.32 & 0.36 & 0.57 & 0.47 \\
\hline 4 & 0.34 & 0.57 & 0.46 & 0.32 & 0.56 & 0.44 & 0.26 & 0.37 & 0.32 & 0.35 & 0.59 & 0.47 \\
\hline 5 & 0.43 & 0.54 & 0.49 & 0.30 & 0.55 & 0.43 & 0.29 & 0.36 & 0.33 & 0.38 & 0.62 & 0.50 \\
\hline 6 & 0.34 & 0.51 & 0.43 & 0.29 & 0.55 & 0.42 & 0.29 & 0.34 & 0.31 & 0.38 & 0.58 & 0.48 \\
\hline 7 & 0.27 & 0.43 & 0.35 & 0.30 & 0.50 & 0.40 & 0.28 & 0.26 & 0.27 & 0.35 & 0.48 & 0.42 \\
\hline 8 & 0.27 & 0.36 & 0.31 & 0.28 & 0.43 & 0.35 & 0.27 & 0.14 & 0.20 & 0.32 & 0.36 & 0.34 \\
\hline 9 & 0.21 & 0.23 & 0.22 & 0.19 & 0.19 & 0.19 & 0.22 & 0.01 & 0.12 & 0.26 & 0.21 & 0.24 \\
\hline 10 & 0.10 & 0.06 & 0.08 & 0.10 & 0.02 & 0.06 & 0.09 & 0.00 & 0.05 & 0.09 & 0.03 & 0.06 \\
\hline average & 0.30 & 0.45 & 0.38 & 0.28 & 0.43 & 0.36 & 0.25 & 0.26 & 0.25 & 0.32 & 0.45 & 0.38 \\
\hline
\end{tabular}

Table 2:

Unconditional cooperation levels within each periods across supergames 1-5, 6-10 and all supergames, respectively for each treatment. Bold numbers indicate the higher cooperation level when comparing the first 5 supergames with the last 5 supergames.

We compare behavior of the first 5 supergames with those in the last 5 supergames. The cooperation levels up to period 5 of supergames 6 to 10 is higher than in the same periods of the first 5 supergame in all the 9 sessions (significant at a $2 \%$ level; for period 6 
and 7 it is still significant on a $10 \%$, using binominal test). Thus, players have to learn to cooperate. On the other hand, cooperation levels in period 9 (7 out 9 sessions, significant at the $10 \%$ level) and period 10 (in all 9 sessions) are lower in the later supergames than in the first supergames which shows that players learn the endeffect: to defect or not to match. ${ }^{10}$

The robustness of the multiple game technique was also found by Bolton et al. (1998) who observe that in dictator games, pooled over all subjects, the same average fraction is given to a single receiver as when faced with several receivers. As in our experiments, the behavior of a subject in the multiple receiver case, however, can be very heterogenous. ${ }^{11}$

\section{Conclusion}

The present experiments have shown that the possibility to choose a partner increases cooperation levels in the finitely repeated prisoner's dilemma. We find that unilateral choice is the best setup. It leads to low defection levels and less singles than with mutual choice. If mutual agreement is necessary cooperation might not arise because a potential cooperator has a lower chance to signal his cooperative intentions. If the potential partner does not want to play, the possibility of future cooperation is foregone.

Our unilateral choice setup allowed us to study players' intentions in the PD game when they choose the outside option by examining their behavior in undesired matches. We were able to show that intending defectors are more likely to exit the game than intending cooperators. While the present experiments study repeated games, the unilateral choice setup might also be interesting for the one-shot game. In the one-shot game player's exit decisions are not caused by past experience and it is therefore the natural environment to study whether people tend to project their own intentions onto others (false consensus idea) as suggested by Orbell et al. (1984) and (1993). By studying the mutual choice setup, these papers could not directly observe players' intentions when no match occurred. Studying the unilateral choice setup would correct for this shortcoming and allow a better understanding whether or not the false consensus idea is valid. If it were valid, moderate cooperators, i.e. players who cooperate sometimes but not always,

\footnotetext{
${ }^{10}$ Selten and Stoecker (1987) use a more sophisticated method to show that players learn to play the endeffect. They examine each subject's choice in the final periods and observe that for each subject both the actual and intended endeffect period move to earlier periods over supergames. Hauk (1997) reports very similar results for our PD and refusal PD setup. Here, we only use an aggregate measure that we believe to be sufficient to show the similarity of our results to Selten and Stoecker's results.

${ }^{11}$ About half of the population gives sometimes more and sometimes less to the single receivers they face. The other half of the subjects never give anything.
} 
would have an evolutionary advantage even in the one-shot game with exit. ${ }^{12}$

Pre-partner selection might be a reason why cooperation levels in real world prisoner's dilemma are surprisingly high. Nevertheless, it cannot be the only reason, since experimental subjects also learn to cooperate when they are forced to play as has been shown in many experiments on the finitely repeated PD game. From a theoretical point of view, no cooperation should arise, unless players expect to meet an altruistic or irrational opponent. Kreps et al. (1982) have shown that if there is a small chance to meet an irrational or altruistic player (a cooperator) it might be in a player's interest to cooperate during several periods. In that case, cooperation would be rational. Thanks to the use of the multiple game technique, we have learnt that most experimental subjects behave probabilistically. Once some cooperation is observed, it is indeed rational to cooperate. Subjects' initial probabilistic behavior might therefore explain cooperation in the finitely repeated $\mathrm{PD}$ game.

The multiple game technique was an important tool in our analysis and we expect it to be an important tool in future experiments, since it seems to be a reliable way to capture probabilistic behavior. At least in the present experiments, aggregate behavior in the multiple game setup was indistinguishable from the behavior in a single game setup. This means that our results are insensitive to the use of multiple games. If this were true in general, the multiple game technique will be an important tool for testing if experimental subjects play mixed equilibria.

\section{References}

Andreoni, J. and Miller, J.H. (1993), "Rational Cooperation in the Finitely Repeated Prisoner's Dilemma: Experimental Evidence", Economic Journal 103, 570585

Ashlock, D., Smucker, S., Stanley, A. And Tesfatsion, L. (1996), "Preferential Partner Selection in an Evolutionary Study of the Prisoner's Dilemma", BioSystems 37, 99-125

Bolton, G.E., Katok, E. And Zwick, R. (1998), "Dictator game giving: Rules of fairness versus acts of kindness", International Journal of Game Theory 27, 269-300

Colomer, J. M. (2000), "Exit, Voice, and Hostility in Cuba", International Migration Review 34(2), 423-442

\footnotetext{
${ }^{12}$ See Orbell et al. (1991), (1996) and Morikawa et al. (1995).
} 
Ghosh, P. And Ray, R. (1996), "Cooperation in Community Interaction without Information Flow", Review of Economic Studies 63, 491-519

Hauk, E. (1997) "Breaking Out: Essays on Partner Selection in Prisoner's Dilemma Games", PhD Thesis, European University Institute, Florence

Hauk, E. (1999) “Multiple Prisoner's Dilemma games with(out) an outside option: an experimental study", Working Paper 391, Universitat Pompeu Fabra, Barcelona

Hauk, E. (2000), "Leaving the Prison: Permitting Partner Choice and Refusal in Prisoner's Dilemma Games", Computational Economics, forthcoming

Hirschman, A. O. (1970), Exit, Voice, and Loyality. Responses to Decline in Firms, Organizations, and States. Cambridge, Mas. Harvard Univerity Press

Hirschman, A. O. (1993) "Exit, Voice and the Fate of the German Democratic Republic", World Politics 45(2), 173-202

Kreps, D.M., Milgrom, P., Robert, J. and Wilson, R. (1982) "Rational Cooperation in the Finitely Repeated Prisoner's Dilemma", Journal of Economic Theory 27, p. $245-252$

Morikawa, T., Orbell, J.M, and Runde, A.S. (1995) "The Advantage of Being Moderately Cooperative", American Political Science Review 89, 601-611

Ochs, J. (1995) "Games with Unique, Mixed Strategy Equilibria: An Experimental Study", Games and Economic Bahavior 10(1), 202-217

Orbell, J.M, and Dawes, R.M. (1991) “A Cognitive Miser Theory of Cooperators' Advantage", American Political Science Review 85, 515-528

Orbell, J.M, And DaWEs, R.M. (1993) "Social Welfare, Cooperator's Advantage and the Option of Not Playing the Game", American Sociological Review 58, 787-800

Orbell, J.M, Runde, A And Morikawa, T. (1996) "The robustness of cognitively simple judgment in ecologies of Prisoner's Dilemma Games", Biosystems 37, 81-97

Orbell, J.M, Schwartz-Shea, P. and Simmons, R.T. (1984) "Do Cooperators Exit More Readily Than Defectors?", American Political Science Review 78, 147162

PECK, J. R. (1993), "Friendship and the Evolution of Cooperation", Journal of Theoretical Biology 162, 195-228 
Schluessler, R. (1989), "Exit Threats and Cooperation under Anonymity", Journal of Conflict Resolution 33(4), 728-749

Selten, R. And Stoecker, R. (1986). "End Behavior in Sequences of Finite Prisoner's Dilemma Supergames", Journal of Economic Behavior and Organization, 7, $47-70$

Stanley, E.A., Ashlock, D. And Tesfatsion, L. (1994), "Iterated Prisoner's Dilemmas with Choice and Refusal of Partners", in C.Langton, editor, Artificial Life III, Volume 17 of Proceedings Volume, pages 131-175, Addison-Wesley, Reading. MA

Tesfatsion, L. (1997), “A Trade Network Game with Endogenous Partner Selection”, in H.Ammon, B.Rustem, and A.B.Whinston, editors, Computational Approaches to Economic Problems, pages 249-269, Kluwer Academic Press, Dordrecht, The Netherlands.

Tiebout, C.M. (1956), "A pure theory of local expenditures", Journal of Political Economy 64, 416-424

Vanberg, V.J. And Congelton, R.D. (1992), "Rationality, Morality and Exit", American Political Science Review 86(2), 418-431

\section{A Instructions}

\section{A.1 Introduction}

You are about to participate in an experiment about decision making. The instructions are simple and if you follow them carefully, you will be able to earn a considerable amount of money. You will be given some starting capital of 1000 pesetas which you can increase or reduce depending on your activity. Should you go bankrupt, you will not earn anything and of course you will not have to pay anything.

\section{A.2 Duration and setup of a session}

- a session

- a session consists of ten periods

- each session involves seven participants. 
- in a session every participant will be identified by a number out of 1 to 7 which is randomly chosen.

- you will keep this identity for the 10 periods of the entire session.

- decisions

In each period you are asked to make two decisions.

1. Your first decision is with whom of the other participants you would like to interact in a two person decision problem. This can be done with 0 to maximally 6 participants. Everybody will make this decision simultaneously.

After everybody has completed the first decision you will be told with whom you are matched. A match occurs between two people if both have chosen to interact with each other. Thus you can have 0 to 6 matches.

2. your second decision is to chose an action for every match you are having.

- in each case you have two possible actions $a$ and $b$. So does the other.

- both of you decide simultaneously which action to choose without knowing the decision of the other.

- Given that you can only choose between $a$ and $b$ there are four possible combinations of actions. Depending on the combination that occurs, you and the other person will get the following points for a match according to the table below.

\begin{tabular}{|c|c|c|c|}
\hline your decision & his decision & your points & his points \\
\hline \hline$a$ & $a$ & -1 & -1 \\
\hline$a$ & $b$ & 7 & -6 \\
\hline$b$ & $a$ & -6 & 7 \\
\hline$b$ & $b$ & 5 & 5 \\
\hline
\end{tabular}


The table is interpreted as follows

$*$ if you chose $a$ and your match chose $a$, you will get -1 points and your match gets -1 points

* if you chose $a$ and your match chose $b$, you will get 7 points and your match will get -6 points

* if you chose $b$ and your match chose $a$, you will get -6 points and your match will get 7 points

* if you chose $b$ and your match chose $b$, you will get 5 points and your match will get 5 points

* if you were not matched with a particular participant, you get zero points and so does he.

\section{A.3 Repetition of a session}

Each of you will be given a number which identifies you over a session (10 periods). In these ten periods you have to make the decisions described above. Once the ten periods are over, you will get a new number, chosen randomly, which identifies you over a second session of ten periods where you will repeat the above procedure. In total there will be 10 sessions of 10 periods with a new identification number in each session.

\section{A.4 Earnings}

The 10 sessions are split into two groups of five sessions. The total payoff you will receive is calculated as follows:

1. Two sessions will be chosen at random, one out of each of the two groups mentioned above. The probability that a session is selected given the group to which it belongs is $\frac{1}{5}$. Thus it is in your interest to do well in all and each single session because two of them will be chosen to calculate your final payoff.

2. The points you obtained in the chosen sessions will be converted into pesetas. One point is equivalent to 15 pesetas. Thus, e.g. 50 points equal 750 pesetas.

3. Your total payoff will be the payoff(loss) from the two sessions chosen plus the fixed starting captial (1000 pesetas). 


\section{A.5 The use of the computer}

\section{A.5.1 The two decision problems}

\section{Partner selection}

For your first decision the computer will display a screen asking you to indicate with whom you would like to interact. It also communicates you your identification number, the current period and your total points in the session so far. You have to indicate using " $y$ " for yes and "n" for no if you want the match. Here is an example:

$$
\begin{array}{lllllll}
1 & 2 & 3 & 4 & 5 & 6 & 7
\end{array}
$$

offer $\mathrm{x}$

The $\mathrm{x}$ under 1 means that you are participant 1 . As you cannot be matched with yourself, there is no empty space below the 1 . In the line "offer" you have to answer whether you want a match or not. To input your answer you have to jump to the field you want to modify using the arrows of the cursor. Once you are on the field, press ENTER, write your answer - $y$ or $n$ - and press ENTER again. Notice that you have to press ENTER twice.

Once you have made all decisions press F10 to communicate them. Once F10 is pressed, your decisions cannot be revised anymore. So make sure you have written what you wanted to write before pressing F10.

\section{Choice of an action}

Any time you have to choose an action the computer will display the table of points that the different combination of actions yield. It will also tell you with whom you are matched using the structure of the following table

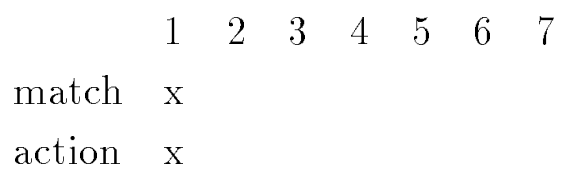

Again you are participant 1 in this example. The line "match" indicates with whom you got matched. If an "n" occurs this means that you are not matched with this participant. If a "y" occurs you have to fill in the empty space in the "action" line by chosing action $a$ or $b$. The following table displays all possible combinations that can occur

$$
\begin{aligned}
& \begin{array}{lll}
1 & 2 & 3
\end{array} \\
& \text { match } x \quad n \quad y \\
& \text { action } \mathrm{x} x
\end{aligned}
$$


ENTER, enter your decision and press ENTER again. You can use different actions in different matches. Once you have made all decisions press F10 to continue the experiment.

Notice that you have to press F10 as well when you are not asked to make any decisions as no match has occurred. Do not forget to do so or you will keep everybody else waiting.

\section{A.5.2 The history}

After every period you will be told your payoffs in that period and you will be shown the history of the experiment. The following table will teach you all possible results that can be registered in the history and how to interpret them.

\begin{tabular}{|c|c|c|c|c|c|c|c|c|}
\hline per & & 1 & 2 & 3 & 4 & 5 & 6 & 7 \\
\hline 1 & $\begin{array}{l}o \backslash \mathrm{s} \\
\text { pay }\end{array}$ & $\begin{array}{c}x \times x \\
00\end{array}$ & $\begin{array}{c}\mathrm{n} \times \mathrm{x} \\
0\end{array}$ & $\begin{array}{c}\mathrm{y} \times \mathrm{x} \\
00\end{array}$ & $\begin{array}{c}\text { y a a } \\
\mathrm{d} \mathrm{d}\end{array}$ & $\begin{array}{c}y \text { a b } \\
\text { h } 1\end{array}$ & $\begin{array}{c}\mathrm{y} \mathrm{b} a \\
1 \mathrm{~h}\end{array}$ & $\begin{array}{c}\mathrm{y} b \mathrm{~b} \\
\mathrm{c} \mathrm{c}\end{array}$ \\
\hline
\end{tabular}

"per" stands for period, "o" for offer "s" for action chosen and "pay" for the points received. You are again participant 1. Behind o\s you are told whether you made an offer(ybackslashn), your own action and your partner's action. An x indicates that no match took place with this participant. "pay" tells you the points you and your partner received in a match. They are zero if you were not matched.

In the first three cases you are not matched, first because you cannot be matched with yourself, secondly because you did not want the match and thirdly because the other did not want the match.

In the remaining four cases you are matched. They just reproduce the content of the points table.

You are able to look into the history any time you have to make a decision and while waiting for the others to make their decisions. You just have to press the corresponding keys. F1 will show you the history of the first session, F2 of the second and F3 of the third, F4 of the fourth, F5 of the fifth, F6 of the sixth, F7 of the seventh, F8 of the eighth, F9 of the nineth and $\mathbf{q}$ of the tenth. If you look into the history while waiting, you will not be aware when the others have finished. So please do not just keep the history window open if you are not consulting it. Notice that if you want to look into the history while waiting the computer reacts fairly slowly. After you pressed F1 or F2 or F3 or F4 or F5 or F6 or F7 or F8 or F9 or q, it will take some seconds before the history window appears. 